\title{
Repetition strengthens target recognition but impairs similar lure discrimination: evidence for trace competition
}

\author{
Zachariah M. Reagh ${ }^{1,2,3}$ and Michael A. Yassa ${ }^{1,2,3,4,5}$ \\ ${ }^{1}$ Department of Neurobiology and Behavior, University of California, Irvine, California 92697, USA; ${ }^{2}$ Center for Neurobiology of \\ Learning and Memory, University of California, Irvine, California 92697, USA; Institute for Memory Impairments and Neurological \\ Disorders, University of California, Irvine, California 92697, USA; ${ }^{4}$ Department of Psychological and Brain Sciences, The Johns Hopkins \\ University, Baltimore, Maryland 21218-2686, USA
}

\begin{abstract}
Most theories of memory assume that representations are strengthened with repetition. We recently proposed Competitive Trace Theory, building on the hippocampus' powerful capacity to orthogonalize inputs into distinct outputs. We hypothesized that repetition elicits a similar but nonidentical memory trace, and that contextual details of traces may compete for representation over time. We designed a task in which objects were incidentally encoded either one or three times. Supporting our theory, repetition improved target recognition, but impaired rejection of similar lures. This suggests that, in contrast to past beliefs, repetition may reduce the fidelity of memory representations.
\end{abstract}

A great deal of neuroscientific and psychological research has been aimed at understanding the way in which memories are encoded and retrieved. Since the seminal studies of amnesic patient H.M. (Corkin 2002; Squire 2009), the critical importance of the hippocampus and surrounding medial temporal cortical regions has been repeatedly demonstrated. However, despite decades of advancements in our understanding of this neural network, there is still much debate about the nature of memories and the role of the hippocampus in supporting them.

The two dominant accounts of the role of the hippocampus are the Standard Model of Systems Consolidation (SMSC) and Multiple Trace Theory (MTT). Both theories assume the necessity of the hippocampus for the encoding of new memories, and rely on a form of hippocampal indexing as a means of interacting with the neocortex (Teyler and DiScenna 1986; Teyler and Rudy 2007). However, these accounts differ in their treatment of the role of the hippocampus during retrieval. SMSC argues that, over time, the hippocampus essentially "teaches" the neocortex which elements are to be bound in support of a memory trace (Squire and Alvarez 1995). Once the neocortex has sufficiently "learned" the memory trace, the hippocampus is no longer assumed to be necessary to support it. On the other hand, MTT proposes that every time a memory is retrieved, a different trace is established, and that the number of these traces corresponds to the strength of that memory (Nadel and Moscovitch 1997). Importantly, MTT distinguishes between semantic and episodic information, and argues that the hippocampus is always necessary to retrieve context-rich episodic information, irrespective of age. Over the years, evidence has accumulated in support of both SMSC (Kim and Fanselow 1992; Maren et al. 1997; Anagnostaras et al. 1999) and MTT (Lehmann et al. 2007; Sutherland et al. 2008).

A more recent theory dubbed the Transformation Hypothesis (Winocur et al. 2007, 2010) discusses a transfer of memories from episodic, contextual content to schematic, semantic content in an attempt at harmonizing between these discrepant findings.

\footnotetext{
${ }^{5}$ Corresponding author E-mail myassa@uci.edu Article is online at http://www.learnmem.org/cgi/doi/10.1101//m.034546.114.
}

Under this view, memories begin as episodic and hippocampally dependent, and are transformed into semantic memories that are represented in extrahippocampal cortices which are not dependent on the hippocampus. That is, the Transformation Hypothesis assumes that the hippocampus' role in memories is dependent on their age such that older and more semantic memories no longer depend on the hippocampus for retrieval. This is in some ways consistent with both SMSC and MTT, as discussed above.

Importantly, SMSC, MTT, and the Transformation Hypothesis do not deal explicitly with the issue of memory interference. This refers to the phenomenon of certain items in memory having some degree of representational overlap, which can make the items in question difficult to distinguish from one another. Inability to overcome this type of interference can lead to ambiguity in a memory trace, and even false remembering. An accumulating body of research examining hippocampal computations has found powerful and intricate structural and functional properties that seem to be especially suited for overcoming this interference. This process of orthogonalizing overlapping inputs has come to be known as pattern separation (Marr 1971; O'Reilly and McClelland 1994; Treves and Rolls 1994), and is thought to be instantiated through sparse firing patterns of dentate gyrus granule cells onto large populations of pyramidal cells in subregion CA3 (Leutgeb et al. 2007; Norman 2010; Yassa and Stark 2011). Studies in rodents (Gilbert et al. 2001; Leutgeb et al. 2007; McHugh et al. 2007) and humans (Bakker et al. 2008; Lacy et al. 2011) have indicated that even with slight deviations from a previously encountered stimulus or context, the dentate gyrus and area CA3 can show evidence for detecting and representing change from an existing representation in memory. The powerful implication of this research is that a process such as pattern separation may underlie the brain's capacity to create the many unique memory traces that together comprise one's personal

\footnotetext{
(C) 2014 Reagh and Yassa This article is distributed exclusively by Cold Spring Harbor Laboratory Press for the first 12 months after the full-issue publication date (see http://learnmem.cshlp.org/site/misc/terms.xhtml). After 12 months, it is available under a Creative Commons License (AttributionNonCommercial 4.0 International), as described at http://creativecommons. org/licenses/by-nc/4.0/.
} 
narrative. As such, memory interference and the consequences of fundamental hippocampal functions suited toward orthogonalization of overlapping inputs into distinct memories are important to consider.

We explicitly incorporated these hippocampal computations into a framework of episodic memory function, which we referred to as Competitive Trace Theory, or CTT (Yassa and Reagh 2013). This account builds directly on hippocampal indexing theory (Teyler and DiScenna 1986; Teyler and Rudy 2007) and attempts to harmonize between ideas of both SMSC and MTT, much like the Transformation Hypothesis (Winocur et al. 2010). In short, we argue largely in agreement with MTT that the hippocampus is always necessary to support context-rich episodic details, but we are in agreement with SMSC that cortical learning can support central features of a memory trace with sufficient time and reactivations. Also in line with the Transformation Hypothesis, we envision memory as existing on a continuum from episodic to semantic content. Critically, we differentiate our account from this and other previous theories by arguing that, in addition to the passage of time, reactivation of events can affect where a memory falls on this continuum. We propose that with every reactivation event, competition may be induced by a similar, but not identical, memory trace being established. That is, the hippocampus is ideally suited to distinguish one event from another in memory, but under conditions of identical input, this ability to represent very similar events differently may lead to somewhat dissimilar representations for identical stimuli. The overlapping elements of the trace are assumed to become strengthened and semanticized, leaving a core set of factual elements of a memory. The nonoverlapping elements-rich contextual details that may be highly similar, but are simply not the same as those of prior encoding events-are assumed to compete with one another for inclusion in the memory trace. This competition is thought to occur at the level of the cortex, which does not have an interference minimization scheme, unlike the hippocampus. The two possible consequences of this competition among nonoverlapping elements are (1) forgetting of these contextual details (decontextualization of the memory trace) and (2) insertion of illusory contextual details in any given reactivation (recontextualization of the memory trace).

One simple prediction of CTT is that, given the hippocampus' powerful capacity for orthogonalization, it is possible that repeated encoding events of identical items will establish these competing elements of memory traces. Though stimulus identity is held constant, external (e.g., certain aspects of an object that are encoded) and internal (e.g., attention and affective fluctuations) contextual elements may vary from one experience to the next. That is, a separate encoding event with an identical central stimulus may lead the hippocampus to create a memory trace with distinct components. This describes a potential negative consequence of pattern separation having occurred. Whereas pattern separation computations reduce interference among similar inputs, it is possible that these computations can create partially nonoverlapping representations of identical stimuli, which would itself induce interference. This would induce competition each time a partially nonoverlapping memory trace is encoded, strengthening overlapping elements (e.g., the sort of object repeatedly seen), and weakening nonoverlapping elements (e.g., very specific details about that object across encounters). We hypothesize asymmetrical consequences of this competition among memory traces: (1) general recognition of studied items should improve with multiple exposures, but (2) detailed discrimination of studied items may actually be hindered by multiple exposures.

We designed a behavioral paradigm to directly test this prediction. Twenty-one healthy volunteers (12 females and nine males, mean age $=20.9 \mathrm{yr}$, standard deviation $=1.3$ ) from the
Johns Hopkins University undergraduate community gave informed consent in accordance with the University's Institutional Review Board, and participated in the study. In this experiment, participants first underwent an incidental encoding phase in which pictures of common objects were judged as being more commonly found "indoors" or "outdoors." Each object was viewed for $2.5 \mathrm{sec}$, with a 0.5 -sec inter-stimulus interval. Responses were made and recorded during the 2.5 -sec stimulus presentation. We manipulated the number of times study items were encountered such that 150 objects were seen only once, and 150 objects were seen a total of three times each (resulting in 600 trials in total) (Fig. 1A). Trial order was pseudorandomized such that items repeated multiple times were not presented back-to-back. Following incidental encoding, subjects completed a surprise retrieval phase in which their memory for the previously seen objects was tested. This phase consisted of 400 objects, 150 of which were repeated targets, 150 were similar lures, and 100 were novel Foils (Fig. 1B). Of the 150 targets and lures, each respectively consisted of 75 objects that were viewed only once during encoding, and the other 75 were viewed three times during encoding. Finally, lure items were categorized into five similarity bins based on a priori behavioral assessments (Lacy et al. 2011). Lure bins ranged from bin 1 to bin 5, with bin 1 being the most similar to the original object, and bin 5 being the most dissimilar. This provided a range of lure similarity, and thus allowed us to analyze the effects of item repetition on discrimination across five levels of difficulty. This aspect of our manipulation is critical, as the amount of memory interference is parametrically distributed across our lure items. This allows us to assess whether any effects of repeated exposures are limited to certain levels of memory interference. In the retrieval phase, subjects were tasked with indicating whether each object was "old" (an identical target) or "new" (a similar lure or a novel foil). The experiment was programmed using PsychoPy stimulus presentation software (Peirce 2007, 2008).

Raw response proportions can be seen in Table 1. For statistical comparisons, target recognition was assessed in terms of normalized $d^{\prime}$ values (z[Target Hit Rate] - z[Foil False Alarm Rate])

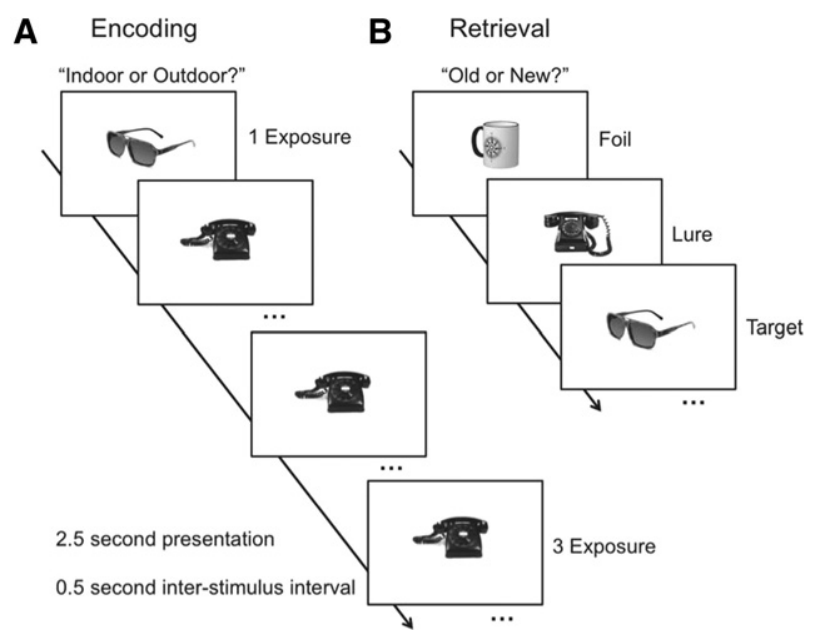

Figure 1. Schematic of the trial design. All images were presented for $2.5 \mathrm{sec}$, with a 0.5 -sec inter-stimulus interval. ( $A$ ) Encoding phase consisting of 600 trials. Judgments were incidental ("Indoor/Outdoor?"), and subjects were unaware of a subsequent memory test. One-hundred-andfifty images were encountered once, and another 150 were encountered three times. (B) Retrieval phase consisting of 400 trials (150 repeated Targets, 150 similar Lures, and 100 novel Foils). Of the Targets and Lures, half of the original images were presented once, and the remaining half three times. Judgments were overtly mnemonic ("Old/New?"). 
Table 1. Raw proportions correct (mean, SD) of target hits and lure correct rejections by number of exposures (mean novel foil $C R$ rate $=0.97, S D=0.04$ )

\begin{tabular}{lcccccc}
\hline & Target hit & $\begin{array}{c}\text { Lure bin } \\
\text { 1 CR }\end{array}$ & $\begin{array}{c}\text { Lure bin } \\
\text { 2 CR }\end{array}$ & $\begin{array}{c}\text { Lure bin } \\
\text { 3 CR }\end{array}$ & $\begin{array}{c}\text { Lure bin } \\
\text { 4 CR }\end{array}$ & $\begin{array}{c}\text { Lure bin } \\
\text { 5 CR }\end{array}$ \\
\hline 1 Exp. & $0.73(0.07)$ & $0.47(0.14)$ & $0.72(0.11)$ & $0.80(0.10)$ & $0.85(0.14)$ & $0.92(0.10)$ \\
3 Exp. & $0.90(0.07)$ & $0.47(0.17)$ & $0.61(0.17)$ & $0.70(0.12)$ & $0.80(0.18)$ & $0.90(0.09)$ \\
\hline
\end{tabular}

fail, and when it is especially low, it may not be required. We additionally note that our null effect at bin 1 is possibly due to floor performance, and that discrimination at this level of interference is maximally difficult (indeed, correct rejection rates here did not differ from chance with either 1 or 3 exposures). Taken together, in the present data,

derived from signal detection analysis. An independent samples $t$-test revealed that subjects performed significantly better at target recognition (assessed via $d^{\prime}$ ) for objects seen three times than for those seen only once $\left(t_{(20)}=3.899, P<0.001\right)$ (Fig. 2A). We note that the average response criterion was not significantly altered between 1- and 3-exposure images across lure bins $\left(\mathrm{C}_{1 \text { exposure }}=0.64, \mathrm{C}_{3 \text { exposures }}=0.69 ; t_{(20)}=0.23, P>0.05\right)$ or within particular lure bins (all $P>0.05$ ), suggesting that the data reflect effects on mnemonic processes rather than simply biased response criteria. This result supports our first hypothesis that general recognition of repeated target items is improved by multiple encoding events.

We assessed discrimination of similar lure objects via a repeated measures ANOVA with number of repetitions (one vs. three) and lure bin (bin 1 through bin 5) as fixed factors. We corrected for response bias by calculating a lure discrimination index (LDI) for each subject: $\mathrm{p}$ ("New"|Lure) - p("New"|Target), or lure correct rejections - target misses. Importantly, we used a pooled term for misses, combining miss rates across 1- and 3-exposure targets. This yields a single value for correcting lure discrimination values, capturing a general tendency toward biased "New" responses for each subject throughout the experiment. Although prior studies in this vein have included a "Similar" response to disambiguate explicit recognition of a lure item vs. simple forgetting of the original stimulus (vs. the more inclusive "New" response), we note that our corrected LDI accounts and corrects for overall forgetting rates. The repeated measures ANOVA revealed a significant effect of lure similarity bin $\left(F_{(4,76)}=88.018, P<0.001\right.$ corrected, $\left.\eta_{P}^{2}=0.822\right)$, indicating variability in difficulty across bins. We furthermore observed a significant effect of the number of exposures $\left(F_{(1,19)}=11.673, P=0.003\right.$ corrected, $\left.\eta_{P}^{2}=0.381\right)$ and a significant interaction between lure bin and number of exposures $\left(F_{(4,76)}=3.267, P=0.026\right.$ corrected, $\left.\eta_{P}^{2}=0.147\right)$. For all observed effects, power $>0.9$. These results suggest that subjects' ability to correctly reject lures varied as a function of exposures, and that this did not affect all lure bins uniformly. Post hoc contrasts were conducted following the ANOVA to probe for differences at specific levels of mnemonic interference across lure bins, which were corrected for multiple comparisons using Scheffé's method (yielding critical $F_{\mathrm{S}}=9.968$ ), and nonsphericity of error. We found that rejection of similar lures was diminished for objects encoded three times relative to those encoded only once in bin $2\left(F_{(1,76)}=13.399, P<0.05\right.$ corrected $)$ and bin 3 $\left(F_{(1,76)}=19.507, P<0.05\right.$ corrected) (Fig. $\left.2 \mathrm{~B}\right)$. Additionally, this same contrast was marginal in lure bin 4 , but fell short of significance given our corrected threshold $\left(F_{(1,76)}=6.214\right.$, uncorrected $P=0.015$ ). It is possible that this particular comparison would have been significant given a larger sample size, although our analyses were clearly powered for detecting effects in other bins (based on observed power $>0.9$ in the ANOVA main effects and interaction). No differences were observed in bins 1 and 5 . In summation, lure discrimination was impaired with multiple exposures in conditions of moderate mnemonic interference (bins 2 and 3, and perhaps bin 4). However, when interference was especially high (bin 1) or low (bin 5), multiple encoding events did not adversely affect mnemonic discrimination. It is important to note that when interference is especially high, pattern separation may bins 2-4 best approximate the trials on which a pattern separation mechanism is likely engaged, although we cannot speak to this directly without supporting neural data.

In this experiment, we presented subjects with common objects that were incidentally encoded, and later tested their ability to recognize target items and correctly reject similar lures. We varied lure similarity to assess the levels of difficulty at which this effect was most pronounced. However, unlike prior studies in this vein, we manipulated the number of times subjects encountered the objects during encoding, with half being viewed only once and the other half being viewed three times. Consistent with our hypotheses based on the recently proposed CTT, we found that target recognition was improved with multiple exposures, but that multiple exposures also degraded discrimination of similar lures from items in memory.

The first result is certainly uncontroversial. It is rather intuitive that multiple opportunities to experience something should strengthen its representation in memory (Ebbinghaus 1885), which is exactly what we observed for target recognition in this experiment. To evoke an example from daily life, one might expect that the ability to recognize a particular intersection of city streets improves as one visits that intersection more frequently.
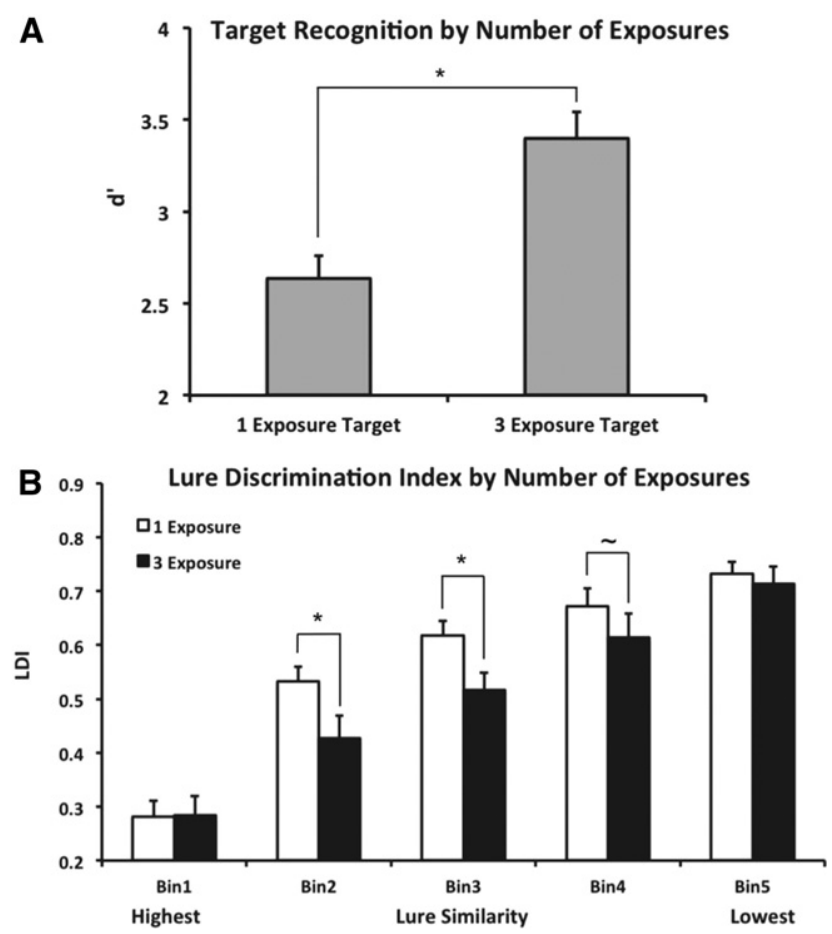

Figure 2. Results from the Retrieval phase. $(A)$ Target recognition performance (normalized $d^{\prime}$ indices) was improved for objects encoded three times compared to those encoded only once. $(B)$ Lure correct rejection (bias-corrected Lure Discrimination Index) was generally hindered by three encoding events compared to a single encoding event. This effect was specific to moderate degrees of mnemonic interference (similarity bins 2 and 3, and marginally in bin 4). 
However, our second result is considerably less intuitive. Our findings suggest that although the ability to generally recognize something is strengthened with multiple encounters, one's ability to discriminate among similar items in memory decays, as evinced by a marked decrease in lure discrimination. Revisiting the example of the intersection of city streets, this implies that if one were to frequently visit a particular intersection, one's ability to finely discriminate that intersection from other very similar ones is not enhanced, but rather hindered. It is possible that such a dynamic involves a trade-off between "gist" memory and "detail" memory (Nadel et al. 2000), respectively enhancing the former and degrading the latter, though the exact mechanistic underpinnings of such a distinction are unclear.

We propose that the mechanism of such a trade-off lies in the capacity of the hippocampus to orthogonalize highly overlapping inputs into orthogonal outputs (Yassa and Stark 2011). This capacity may be so robust, in fact, that identical repetitions are nonetheless capable of inducing competition among memory traces that can enhance the overlapping central features of a memory, but lead to drop-off of distinguishing contextual details as recently proposed by CTT (Yassa and Reagh 2013). This notion is consistent with recent evidence that the extent of similarity among neural patterns from one exposure to the next is predictive of subsequent memory (Xue et al. 2010), and it stands to reason that even exact repetitions of a studied item may, in some cases, lead to a decay in such pattern similarity. In our own model, we have conceived of memory traces existing on a continuum from richly detailed and episodic to decontextualized and semantic, with the passage of time and the occurrence of reactivation events driving the process (Fig. 3). The present data support this account of memory updating. Alternatively, repetition has been observed to shift reliance toward familiarity (rather than recollection) as one remembers a given event (Jacoby et al. 1998). As such, repetition may affect one's ability to discriminate as a memory trace shifts its dependence entirely from the hippocampus to cortical substrates, and may elicit differential effects of decay and interference. In particular, higher reliance on familiarity may make a memory trace more susceptible to interference, and may thus hinder discriminability (Sadeh et al. 2014). We note, however, that such an account is not inconsistent with the tenets of CTT, and that increased reliance on familiarity can be seen as a result of semanticization as the hippocampus itself induces interference with repetition.

A limitation of this experiment lies in the range of the number of exposures used. Although we could certainly extend this range to include conditions of 5 or more exposures, this would lead the encoding phase of the task to be prohibitively long in duration. Also, in preliminary work, we discovered that if addi-

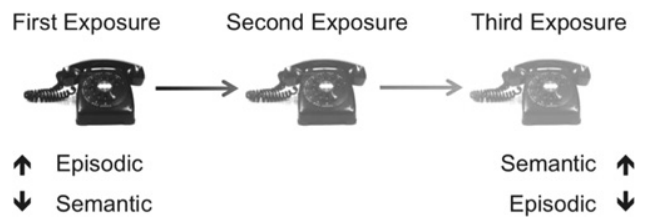

Figure 3. Conceptual illustration of the altered fidelity of an item in memory with multiple repetitions. Competitive Trace Theory considers information in memory on a continuum from a memory trace rich in episodic, contextual detail to decontextualized, semanticized trace. The theory assumes that the passage of time leads to such a transfer function, and that reactivations of a particular trace (even if an identical item is presented) can elicit unique contextual elements from one encoding event to the next, inducing competition among those elements and speeding the process. As per this example, the semantic knowledge of having seen a phone is strengthened, but the episodic details of what that particular phone looked like are degraded. tional encoding opportunities are introduced, subjects become keenly aware of the repetition, and the nature of the task changes. Specifically, with five repetitions, subjects became aware of the repetition, and reported engaging mnemonic strategies (counting exposures, comparison and matching to last exposure, etc.). In the three-exposures condition used here, some subjects reported being somewhat aware of multiple exposures to certain stimuli, but did not recognize the extent to which images were being repeated. Thus, we opted to use the 3-exposure condition.

We note that the timeframe of the present study differs from those described by consolidation theories. Specifically, our experiment operates over the order of an hour or so, whereas consolidation is thought to take substantially longer in the mammalian brain. It is likely that the consolidation window is quite variable and that some of these synaptic modification processes begin on a smaller timescale. For example the conversion of early to late long-term potentiation occurs over the first hour after encoding an experience (Frey and Morris 1997). During that window, it is possible that reactivation of the memory allows for synaptic modification. This time window is also consistent with reconsolidation studies (Tronson and Taylor 2007). Importantly, a key tenet of CTT is that, in addition to the passage of time, reactivations can cause a memory to become decontextualized and lose episodic detail. It is this latter component that our experiment specifically manipulated, though in future experiments we intend to test the predictions of CTT as relates to elapsed time, and its interaction with reactivations.

These data provide basic support for one of the central tenets of CTT and offer a simple paradigm by which trace competition can be assessed in future studies using high-resolution brain imaging of hippocampal subfields.

\section{Acknowledgments}

We thank Craig Stark and Gregory Sanchez for contributions to design and data collection in earlier iterations of the experiment. We also thank Liz Murray, Sophia Fleming-Benite, and Natasha $\mathrm{Ng}$ for help with participant recruitment and testing. M.A.Y. is supported by National Institute on Aging grants P50 AG05146 and R01 AG034613. Z.M.R. is supported by National Science Foundation Graduate Research Fellowship (Division of Graduate Education) grant DGE-1232825.

\section{References}

Anagnostaras SG, Maren S, Fanselow MS. 1999. Temporally graded retrograde amnesia of contextual fear after hippocampal damage in rats: within-subjects examination. J Neurosci 19: 1106-1114.

Bakker A, Kirwan CB, Miller M, Stark CEL. 2008. Pattern separation in the human hippocampal CA3 and dentate gyrus. Science 319: 1640-1642. Corkin S. 2002. What's new with the amnesic patient H.M.? Nat Rev Neurosci 3: 153-160.

Ebbinghaus H. 1885. Memory: a contribution to experimental psychology. Von Duckner and Humboldt, Leipzig, Germany.

Frey U, Morris RGM. 1997. Synaptic tagging and long-term potentiation. Nature 385: 533-536.

Gilbert PE, Kesner RP, Lee I. 2001. Dissociating hippocampal subregions: double dissociation between dentate gyrus and CA1. Hippocampus 11: 626-636.

Jacoby LL, Jones TC, Dolan PO. 1998. Two effects of repetition: support for a dual-process model of know judgments and exclusion errors. Psychon Bull Rev 5: 705-709.

Kim JJ, Fanselow MS. 1992. Modality-specific retrograde amnesia of fear. Science 256: 675-677.

Lacy JW, Yassa MA, Stark SM, Muftuler LT, Stark CEL. 2011. Distinct pattern separation related transfer functions in human CA3/dentate and CA1 revealed using high-resolution fMRI and variable mnemonic similarity. Learn Mem 18: $15-18$.

Lehmann H, Lacanilao S, Sutherland RJ. 2007. Complete or partial hippocampal damage produces equivalent retrograde amnesia for remote contextual fear memories. Eur J Neurosci 25: 1278-1286. 
Leutgeb JK, Leutgeb S, Moser M-B, Moser EI. 2007. Pattern separation in the dentate gyrus and CA3 of the hippocampus. Science 315: 961-966.

Maren S, Aharonov G, Fanselow MS. 1997. Neurotoxic lesions of the dorsal hippocampus and Pavlovian fear conditioning in rats. Behav Brain Res 88: $261-274$.

Marr D. 1971. Simple memory: a theory for archicortex. Philos Trans R Soc Lond B Biol Sci 262: 23-81

McHugh TJ, Jones MW, Quinn JJ, Balthasar N, Coppari R, Elmquist JK, Lowell BB, Fanselow MS, Wilson MA, Tonegawa S. 2007. Dentate gyrus NMDA receptors mediate rapid pattern separation in the hippocampal network. Science 317: 94-99.

Nadel L, Moscovitch M. 1997. Memory consolidation, retrograde amnesia and the hippocampal complex. Curr Opin Neurobiol 7: 217-227.

Nadel L, Samsonovich A, Ryan L, Moscovitch M. 2000. Multiple trace theory of human memory: computational, neuroimaging, and neuropsychological results. Hippocampus 10: $352-368$.

Norman KA. 2010. How hippocampus and cortex contribute to recognition memory: revisiting the complementary learning systems model. Hippocampus 20: 1217-1227.

O’Reilly RC, McClelland JL. 1994. Hippocampal conjunctive encoding, storage, and recall: avoiding a trade-off. Hippocampus 4: 661-682.

Peirce JW. 2007. PsychoPy-Psychophysics software in Python. J Neurosci Methods 162: 8-13.

Peirce JW. 2008. Generating stimuli for neuroscience using PsychoPy. Front Neuroinformatics 2: 10 .

Sadeh T, Ozubko JD, Winocur G, Moscovitch M. 2014. How we forget may depend on how we remember. Trends Cogn Sci 18: 26-36.

Squire LR. 2009. The legacy of patient H.M. for neuroscience. Neuron 61: 6-9.

Squire LR, Alvarez P. 1995. Retrograde amnesia and memory consolidation: a neurobiological perspective. Curr Opin Neurobiol 5: 169-177.
Sutherland RJ, O'Brien J, Lehmann H. 2008. Absence of systems consolidation of fear memories after dorsal, ventral, or complete hippocampal damage. Hippocampus 18: 710-718.

Teyler TJ, DiScenna P. 1986. The hippocampal memory indexing theory. Behav Neurosci 100: 147-154.

Teyler TJ, Rudy JW. 2007. The hippocampal indexing theory and episodic memory: updating the index. Hippocampus 1169: $1158-1169$.

Treves A, Rolls ET. 1994. Computational analysis of the role of the hippocampus in memory. Hippocampus 4: 374-391.

Tronson NC, Taylor JR. 2007. Molecular mechanisms of memory reconsolidation. Nat Rev Neurosci 8: 262-275.

Winocur G, Moscovitch M, Sekeres M. 2007. Memory consolidation or transformation: context manipulation and hippocampal representations of memory. Nat Neurosci 10: $555-557$.

Winocur G, Moscovitch M, Bontempi B. 2010. Memory formation and long-term retention in humans and animals: convergence towards a transformation account of hippocampal-neocortical interactions. Neuropsychologia 48: 2339-2356.

Xue G, Dong Q, Chen C, Lu Z, Mumford JA, Poldrack RA. 2010. Greater neural pattern similarity across repetitions is associated with better memory. Science 330: 97-101.

Yassa MA, Reagh ZM. 2013. Competitive Trace Theory (CTT): a role for the hippocampus in contextual interference during retrieval. Front Behav Neurosci 7: 107.

Yassa MA, Stark CEL. 2011. Pattern separation in the hippocampus. Trends Neurosci 34: 515-525.

Received January 23, 2014; accepted in revised form May 8, 2014. 


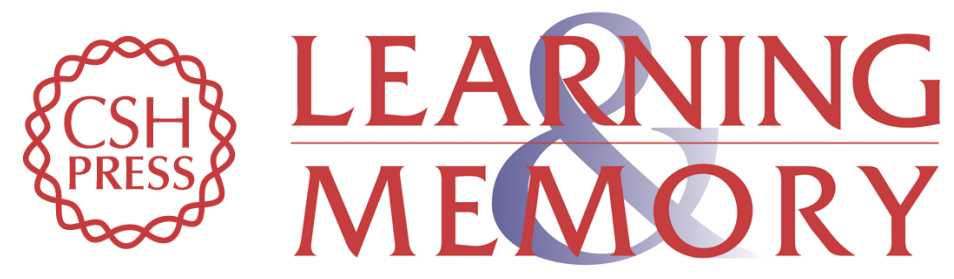

\section{Repetition strengthens target recognition but impairs similar lure discrimination: evidence for trace competition}

Zachariah M. Reagh and Michael A. Yassa

Learn. Mem. 2014, 21:

Access the most recent version at doi:10.1101/Im.034546.114

\begin{aligned} & \hline References $\begin{array}{l}\text { This article cites } 32 \text { articles, } 6 \text { of which can be accessed free at: } \\ \text { http://learnmem.cshlp.org/content/21/7/342.full.html\#ref-list-1 }\end{array} \\ & \begin{array}{r}\text { Creative } \\ \text { Commons } \\ \text { License }\end{array} \begin{array}{l}\text { This article is distributed exclusively by Cold Spring Harbor Laboratory Press for the } \\ \text { first } 12 \text { months after the full-issue publication date (see } \\ \text { http://learnmem.cshlp.org/site/misc/terms.xhtml). After } 12 \text { months, it is available under } \\ \text { a Creative Commons License (Attribution-NonCommercial } 4.0 \text { International), as } \\ \text { described at http://creativecommons.org/licenses/by-nc/4.0/. }\end{array} \\ & \begin{array}{c}\text { Receive free email alerts when new articles cite this article - sign up in the box at the } \\ \text { top right corner of the article or click here. }\end{array} \\ &$\hline\end{aligned}

